\title{
MANAGEMENT OF MULTIPLE CONGESTED CONDITIONS IN UNBUNDLED OPERATION OF A POWER SYSTEM
}

\author{
Hans Glavitsch \\ Swiss Federal Institute of Technology \\ Zurich, Switzerland \\ haglav@eeh.ee.ethz.ch
}

\author{
Fernando Alvarado \\ The University of Wisconsin \\ Madison, Wisconsin, USA \\ alvarado@engr.wisc.edu
}

\begin{abstract}
This paper studies congestion management based on congestion pricing as may be done by an Independent System Operator. Four main concepts are discussed: congestion pricing can lead to the same solution as an Optimal Power Flow, pricing need not have cost information available, good estimates of nonlinear cost coefficients are necessary, and pricing for congestion management is separable from pricing for the purpose of transmission network revenue reconciliation. Emphasis is on the determination of sufficient information by observation of the behavior of the market to permit optimum pricing without explicit communication among parties, and without generators ever being explicitly concerned about the relief of flow congestion. Results from simulated experiments are presented.
\end{abstract}

Keywords: Pricing, congestion, security, Optimum Power Flow, OPF.

\section{Introduction}

The control of electric power grids is about to undergo a major paradigm shift [5], to which this paper contributes by studying price signals as a means to manage congestion in a power system. Price signals for congestion management are not new. They are part of California's plan for unbundled system operation, although it remains a politically charged issue [6]. Congestion pricing is based on concepts from [4]. Two other means for congestion management are quantity-curtailing (used by the National Grid Company [8]), and mandated coordinated trades [9].

Reference [6] concluded that the effectiveness of pricing is questionable because of "gaming" opportunities it affords. This conclusion pertained to the use of congestion pricing as a means for revenue generation in connection with transmission investments. This paper explores pricing only as a congestion management tool, not its role for transmission system revenue generation.

For the sake of explaining the principle, a lossless network is considered. Only constraints associated with a base case are taken into consideration (i.e., no "con-

PE-166-PWRS-16-09-1997 A paper recommended and approved by the IEEE Power System Engineering Committee of the IEEE Power Engineering Society for publication in the IEEE Transactions on Power Systems. Manuscript submitted May 27, 1997; made available for printing September 30, 1997. tingency constraints"). When congestion occurs, it is relieved entirely by re-scheduling of generation. Other means for congestion relief (such as opening of overloaded lines or load shedding) are not explicitly considered. Implicit in this assumption is that price signals are only sent to generators: loads remain inelastic, even though [7] has described the important effect that demand elasticity can have on congestion management ${ }^{1}$.

When price signals are sent to generators to relieve congestion, they are sent as additive (or possibly subtractive) marginal costs which must be paid by (to) the generators to (by) the grid. The effect of these signals is to alter the generation costs, and thus result (under the assumption of rational economic behavior) in a change in the generation dispatch pattern.

The central ideas of this paper are:

- Based on estimates of quadratic cost coefficients around a market solution, operators responsible for congestion management can use price signals to the generators to manage congestion [4].

- System operators need not know the marginal cost of suppliers. They can infer the required parameters from an observation of the market.

- The solution obtained by this process under assumptions of rational economic behavior of all generators is identical to the solution obtained by a centrally dispatched optimum power flow.

- The use of price for congestion relief is separable from its use for transmission revenue generation.

The quadratic component of the supply costs gives information on the rate of change of marginal costs. In other words, quadratic terms predict how marginal costs change as production levels change. Quadratic coefficients have been used previously by several authors, including [3], who assume quadratic benefit functions to design electricity tariffs. The present paper exploits the assumed quadratic property of costs to determine congestion prices which relieve congestion optimally.

Hogan [1] proposed the use of the ideas of [4] for the establishment of a market of tradeable capacity rights. A further elaboration of the idea has been presented in [2], where the capacity rights are not associated with pairs of nodes but instead with the capacity of each individual line. The present work does not use capacity rights directly. Instead, congestion pricing is the primary means for congestion management. Markets on capacity of transmission become indirect: in anticipation of congestion, suppliers of power compete for the

\footnotetext{
${ }^{1}$ The inclusion of responsive demand is trivial if customer benefit curves have the same general response characteristics as the suppliers' costs.
} 
purchase or rights for use of resources in uncongested zones. All trading deals with power, albeit differentiated by location. Trading in transmission is implicit.

\section{Congestion in a vertically organized utility}

A vertically organized power system comprises generation, transmission and distribution, whereby all control functions are within the control of the energy management system. Generation is dispatched in order to achieve the most economic overall solution. In simple control centers, the main tool used for dispatch is economic dispatch control based on the knowledge of the marginal costs of production for each unit in the system. In a more sophisticated environment, the tool of choice is an optimal power flow, which, in addition to its role in minimizing production cost, provides the possibility of avoiding congestion in a minimum cost manner. Congestion management, in this sense, means that a generation pattern is determined such that flow limits are not exceeded. The presence of constraints generally leads to higher marginal costs and reduced revenues. These higher marginal costs and reduced revenues act as an economic signal to the utility. The signal can be used to rearrange generation (for example, to commit or start different units). A persistent congestion problem is an indication to install new generation at certain locations, or to build additional transmission facilities.

\section{Unbundled operation}

Assume that the operation of a power system is unbundled, i.e. generation, transmission, distribution and system control are separate entities in terms of ownership and management. Several options to manage and operate unbundled systems exist [10]. These options vary from "minimal compliance" options, to options where the system is maximally unbundled. Almost all options foresee an Independent System Operator (ISO) to manage system security.

One of the most appealing options in the new environment is where the role of the ISO is limited exclusively to congestion management. In such a structure consumers can choose their supplier, and hence all transactions are in principle bilateral. Among participants an open market is assumed where all transactions are settled, presumably at approximately equal marginal costs. An open market means that all power producers and consumers know each others bids, but not each other costs or production cost functions.

Two premises of the ISO concept as used in this paper are: (1) The ISO need not know prices to perform its function and (2) the ISO imposes and adjusts appropriate congestion charges to attain its objective of assuring coordination among transactions.

Although the ISO need not know the final marginal cost at which the market settles, the ISO assumes that all transactions are agreed upon at equal incremental costs. However, since the ISO is required to coordinate the various transactions, this means that the ISO must be privy to actual and proposed transactions in order that they can be checked for feasibility and security.
The ISO then determines any charges for transmission services (losses, reactive power, reserves, and other network services ${ }^{2}$ ). The present paper deals with the use of pricing to relieve congestion under unbundled operation, as shown below.

\section{Consequences of congestion}

Congestion in a transmission system, whether vertically organized or unbundled, cannot be tolerated, otherwise cascading outages with uncontrolled loss of load will result. Sometimes congestion can be relieved by "cost free" means ${ }^{3}$ such as:

- Outaging of congested lines [12].

- Operation of transformer taps, particularly phase shifters [13].

- Operation of FACTS devices, particularly series devices (including other FACTS-like devices, such as Interphase Power Controllers).

This paper assumes that congestion cannot be alleviated by these means (or rather, that any cost-free means for congestion control have already been exercised).

Not-cost-free means for congestion relief include:

- Re-dispatch of generation in a manner different from the natural settling point of the market. That is, some generators back down while others increase their output. The effect of this is that generators no longer operate at equal incremental costs.

- Curtailment of loads and the exercise of (not-costfree) load interruption options.

This paper restricts its attention to the case where the entire responsibility for congestion relief is on the generation (or supply) side. However, the ideas of this paper can be readily extended to the case where demands can participate in this effort [7].

Congestion inevitably results in an economic surplus loss, irrespective of pricing policies. If the surplus loss is absorbed by the generators (because of fixed demand price policies or because of reluctance to curtail load), the consequence of congestion is higher total production costs, just as in the case of a vertically organized system. However, unlike the vertically organized system case, in the unbundled concept the question arises as to the handling of the extra cost. One approach is to include the extra cost in the charges for transmission services and use signals to power producers having the character of costs to rearrange the power injections such that the congestion is avoided.

Cost signals alter the marginal cost seen by parties. Thus, the market settles into a new equilibrium, presumably arrived at by equalizing the original marginal costs after the constraint cost adder is imposed. This comes about when consumers (or the pool) choose a different generating pattern to minimize costs, and such a pattern happens to avoid the congestion.

\footnotetext{
${ }^{2}$ See [11] for details on pricing some of these other services.

${ }^{3}$ These congestion-relief means are cost-free only to the extent that the marginal cost of using a device is negligible. Investment costs can be substantial.
} 


\section{The role of the OPF and corrective signals}

If all costs are correctly and explicitly disclosed and all constraints enforced, then an Optimum Power Flow (OPF) [14] would indeed find an optimal solution point that meets all constraints ${ }^{4}$. However, in a deregulated environment costs are not explicitly disclosed, at best they are indirectly revealed by the bidding patterns of the participants. As a preamble to the next section, this section assumes that a set of "true marginal costs" exists, and that these costs have been revealed to the operator (possibly in the form of bids). The operator "manages" the system by issuing orders to change dispatch patterns.

Consider a system with $n_{g}$ generators. Let the cost of generator $i$ be:

$$
C_{i}=a_{i}+b_{i} P_{i}+\frac{1}{2} c_{i}\left(P_{i}\right)^{2}
$$

Optimality is determined by defining a Lagrangian and finding its derivative with respect to all the variables:

$$
\begin{aligned}
b_{1}+c_{1} P_{1} & =\lambda \\
b_{2}+c_{2} P_{2} & =\lambda \\
& \vdots \\
b_{n_{g}}+c_{n_{g}} P_{n_{g}} & =\lambda \\
P_{1}+P_{2}+\cdots+P_{n_{g}} & =P_{D}
\end{aligned}
$$

where $P_{D}$ represents total system demand and equation 1 represents the power balance equation for the lossless case. The solution of these equations leads to the following linear matrix problem:

$$
\begin{aligned}
\operatorname{diag}(\mathbf{c}) \cdot \mathbf{P}-\operatorname{ones}\left(n_{g}, 1\right) \cdot \lambda & =-\mathbf{b} \\
\operatorname{ones}\left(1, n_{g}\right) \cdot \mathbf{P} & =P_{D}
\end{aligned}
$$

where $\mathbf{P}$ is a vector of generator powers, $\mathbf{b}$ and $\mathbf{c}$ are cost coefficient vectors, and $\lambda$ is a scalar.

The formulation so far requires only $P_{i}$ and $\lambda$ as the variables of interest. This formulation can be extended to the case of binding congestion by the construction of the injection-to-flow sensitivity matrix $\mathbf{S}$. Let $\mathbf{B}$ be the sparse nodal susceptance matrix (it excludes the reference bus). Let $\mathbf{X}$ be the inverse of $\mathbf{B}$ (only certain entries of $\mathbf{X}$ are needed and they can thus be obtained by sparse inverse techniques). Entries of the injectionto-flow sensitivity matrix are obtained from:

$$
S_{i j, k}=\left(X_{k, i}-X_{k, j}\right) / x_{i j}
$$

where $x_{i j}$ is the reactance of the constrained line in question and $X_{k, i}$ and $X_{k, j}$ are entries of $\mathbf{X}$.

If an unconstrained optimal solution leads to an unfeasible optimum, feasibility can be restored by solving a new problem with the constrained flow(s) added as inequality constraint(s). Under dc power flow assumptions, the constraining line flow (s) are given by:

$$
\mathbf{S} \cdot \mathbf{P} \leq \mathbf{p}^{\max }
$$

where $S$ is the $\left(n_{b} \times n\right)$ matrix of sensitivities of flows to injections, and $\mathbf{p}^{\max }$ is a vector of maximum permissible flow(s) in the binding line(s).

The complete OPF problem formulation leads to the following (still linear) set of conditions:

$$
\begin{aligned}
& \operatorname{diag}(\mathbf{c}) \cdot \mathbf{P}-\operatorname{ones}\left(n_{g}, 1\right) \cdot \lambda+\mathbf{S}^{\mathbf{T}} \cdot \boldsymbol{\mu}=-\mathbf{b} \\
& \operatorname{ones}\left(1, n_{g}\right) \cdot \mathbf{P}=P_{D} \\
& \mathbf{S} \cdot \mathbf{P} \leq \mathbf{p}^{\max } \\
&{ }^{4} \text { Under fixed demand assumptions, an OPF leads to a soci- }
\end{aligned}
$$
etally optimal solution by maximizing surplus.
The marginal production costs are no longer equal, even without losses. However, dispatch of power according to these equations leads to a least cost dispatch.

Additive prices can be determined such that, if the problem is re-optimized ignoring all constraints, the constrained optimal solution is obtained. The determination of a set of (non-unique) values for this additive price vector $\beta$ is:

$$
\beta=-\operatorname{diag}(\mathbf{c}) \cdot \mathbf{P}^{*}+\operatorname{ones}\left(n_{g}, 1\right) \cdot \lambda-\mathbf{b}
$$

where $\mathbf{P}^{*}$ is the solution of the (constrained) optimum power flow with full knowledge of costs. Application of these adder terms $\beta$ leads to the solution of a new unconstrained problem with linear cost terms $\mathbf{b}+\beta$ :

$$
\begin{aligned}
\operatorname{diag}(\mathbf{c}) \cdot \mathbf{P}^{0}-\operatorname{ones}\left(n_{g}, 1\right) \cdot \lambda & =-(\mathbf{b}+\beta) \\
\operatorname{ones}\left(1, n_{g}\right) \cdot \mathbf{P}^{0} & =P_{D}
\end{aligned}
$$

This solution has the property that $\mathbf{P}^{0}=\mathbf{P}^{*}$.

Thus, the following procedure can be used by an ISO that is fully knowledgeable of costs to relieve congestion and attain optimality by pricing:

1 Solve an OPF problem.

2 If constraints result, determine prices $\beta$ that can be sent to the market to relieve the congestion.

3 Issue these prices and observe the market response.

If all assumptions made by the ISO are correct, the resulting operating point should not violate any constraints, and should be a minimum cost solution. In other words, if appropriate additive prices $\beta$ are communicated to the power producers and they follow their economic objective, the free market operating point will be identical to the constrained OPF, i.e. a generation pattern without congestion.

A corollary from this section is that any purely additive cost applied equally to all producers does not have any effect on the ultimate equilibrium point (ignoring demand elasticity as stipulated earlier).

\section{If cost functions are unknown to the ISO}

This section considers the case where the cost functions themselves are unknown to the ISO. The ISO is only able to observe the actual or proposed behavior of the various interacting parties, and must take action based on this behavior. On first thought it may appear that this is not a solvable problem: after all, how is the ISO going to issue prices to elicit behavior change if he/she does not have prices or price information? This section shows that this is indeed possible, albeit it requires at least one iteration between the supplier's bids and the ISO proposed transmission charges.

As indicated above, there is a simple congestion pricing strategy which allows the ISO to interact successfully with the power producers, provided the ISO knows the true costs: run an OPF based on the assumed cost curves, and issue additive prices $\beta$ so that the new equilibrium point occurs "naturally." If the ISO assumptions about the linear cost terms are incorrect, this has no effect on the proposed congestion prices. However, when the ISO does not know the quadratic coefficients around the unconstrained flow pattern this approach 
will not yield the desired result. Four (not mutually exclusive) approaches to the estimation of the quadratic cost coefficients of the generators suggest themselves:

- The operator can require that, although costs need not be revealed, the manner in which costs vary must be revealed. This is undesirable in a competitive environment.

- The ISO can issue him/herself appropriate supplementary quadratic cost components to all producers, consistent with marginal losses. This will likely be necessary, but insufficient.

- Over time, by observing the behavior of generators under a variety of conditions, the ISO infers quadratic coefficient values for all generators. As explained below, this approach is almost (but not quite) sufficient.

- The ISO uses observed responses to transmission congestion charges to estimate the coefficients.

This paper considers further only the third and fourth approaches above. Let $b_{i}$ and $c_{i}$ be the true linear and quadratic cost coefficients for generator $i$. These numbers are unknown to the ISO, but they dictate the behavior of the market. In the absence of price modifiers, the market will settle at an operating point where:

$$
\mathbf{b}+\operatorname{diag}(\mathbf{c}) \cdot \mathbf{P}^{0}=\operatorname{ones}\left(n_{g}, 1\right) \cdot \lambda_{0}
$$

As conditions change, the generation patterns will change, and so will $\lambda$. However, it is assumed that the cost coefficients remain constant. Thus, a second uncongested operating conditions leads to:

$$
\mathbf{b}+\operatorname{diag}(\mathbf{c}) \cdot \mathbf{P}^{1}=\operatorname{ones}\left(n_{g}, 1\right) \cdot \lambda_{1}
$$

Subtracting these last two sets of equations from each other leads to the following equation:

$$
\operatorname{diag}(\mathbf{c}) \cdot \Delta \mathbf{P}=\Delta \lambda \cdot \operatorname{ones}\left(n_{g}, 1\right)
$$

Based on the fact that changes in generation pattern $\Delta \mathbf{P}$ must be according to the ratios of values of $\mathbf{c}$, the ISO can now estimate $c$ values based on observation of $\Delta \mathbf{P}$., Denote these estimates as $\tilde{\mathbf{c}}$. One $\tilde{c}_{i}$ coefficient must be guessed, since it is only the ratio among values that can be observed at this point. Without loss of generality, assume that this is the coefficient for generator 1. The remaining coefficients are estimated from:

$$
\tilde{c}_{2}=\frac{\Delta P_{1}}{\Delta P_{2}} \tilde{c}_{1}, \quad \ldots \quad, \tilde{c}_{n_{g}}=\frac{\Delta P_{1}}{\Delta P_{n_{g}}} \tilde{c}_{1}
$$

A potential problem occurs when $\Delta P_{i}=0$. However, under these circumstances it must be assumed that the particular generator is either unable or unwilling to participate in redispatch, and it must be thus excluded from the list of participating generators and treated as a constant output unit, therefore reducing the dimensionality of the problem ${ }^{5}$. It can be seen further that, if $\Delta \lambda$ were known, the values for $\tilde{c}$ would be known accurately. As it is, these values become known up to a constant. That is:

$$
\tilde{\mathbf{c}}=K \mathbf{c}
$$

where $K$ is a scalar, and $\mathbf{c}$ and $\tilde{\boldsymbol{c}}$ are vectors.

\footnotetext{
${ }^{5}$ The ability of units to adjust their output offers a distinct and measurable economic benefit to the system. Units with fixed output are unable to contribute to this benefit. However, the quantification of the economic benefit offered by units able to adjust their output is beyond the scope of this paper.
}

This process of "almost" estimating the quadratic coefficients by a passive observation of market behavior can be repeated as often as desired, leading to redundant solutions for the estimated values of $\tilde{\mathbf{c}}$. The determination of $\tilde{\mathbf{c}}$ under these circumstances becomes a least squares problem.

The ISO needs also an estimate for the linear cost coefficients $\mathbf{b}$. Denote these estimates by $\tilde{\mathbf{b}}$. These coefficients have an arbitrary adder component, as described above. By assuming an arbitrary $\lambda$ and inserting the observed $P_{i}$ 's in the optimality condition of the unconstrained problem, the ISO receives a set of $\tilde{\mathbf{b}}$ consistent with $\tilde{\mathbf{c}}$. With both these values, the ISO is able to determine corrective price signals as follows.

Assume the ISO detects a single or multiple congestion condition $\mathbf{P}^{3}$ as a result of some outage, anticipated outage, change in demand, or change in proposed generation pattern. An OPF solution, as in the previous section, using a best estimate for the values of $c$ leads to an optimal solution $\widetilde{\mathbf{P}}$, which differs from $\mathbf{P}^{3}$.

It is possible to attain $\widetilde{\mathbf{P}}$ by means of congestion charges. Let $\beta$ be the vector of proposed congestion charges determined as in the previous section:

$$
\begin{aligned}
& K \cdot \mathbf{c} \cdot \widetilde{\mathbf{P}}-\operatorname{ones}\left(n_{g}, 1\right) \cdot \tilde{\lambda}+S^{T} \cdot \underline{\mu}=-\tilde{\mathbf{b}} \\
& \operatorname{ones}\left(1, n_{g}\right) \cdot \widetilde{\mathbf{P}}=P_{D} \\
& \mu \geq 0 \quad \mathbf{S} \cdot \widetilde{\mathbf{P}} \leq \mathbf{p}^{\max }
\end{aligned}
$$

Congestion prices $\beta$ are computed from equation 2 . The ISO sends out these prices to each power producing unit and the market settles into a new proposed operating point $P^{4}$ :

$$
\begin{aligned}
\mathbf{c} \cdot \mathbf{P}^{4}-\operatorname{ones}\left(n_{g}, 1\right) \cdot \lambda_{3} & =-(\tilde{\mathbf{b}}+\beta) \\
\operatorname{ones}\left(1, n_{g}\right) \cdot \mathbf{P}^{4} & =P_{D}
\end{aligned}
$$

Because the cost coefficients may not be correct, however, the solution $\mathbf{P}^{4}$ to which the market settles may be different from the predicted solution $\widetilde{\mathbf{P}}$. The difference is due to the unknown factor $K$. It is possible to determine $K$ from an observation of the difference between the presumed solution change $\Delta \widetilde{\mathbf{P}}=\widetilde{\mathbf{P}}-\mathbf{P}^{3}$ to the observed change $\Delta \mathbf{P}=\mathbf{P}^{4}-\mathbf{P}^{3}$ from:

$$
K=\frac{\Delta P_{i}}{\Delta \widetilde{P}_{i}}
$$

This solution for $K$ is redundant (all $n_{g}$ generators should give the same result). Redundancy is desirable to account for odd producer behavior, response errors and other practical estimation difficulties.

Once $K$ has been estimated, the ISO is able to induce the expected optimal congestion-relieving behavior by issuing a second and final round of additive price signals $\beta^{*}$ obtained from a solution of the new OPF problem:

$$
\begin{aligned}
K \cdot \operatorname{diag}(\tilde{\mathbf{c}}) \cdot \mathbf{P}^{5}-\text { ones }\left(n_{g}, 1\right) \cdot \lambda+\mathbf{S}^{T} \cdot \mu & =-\tilde{\mathbf{b}} \\
\operatorname{ones}\left(1, n_{g}\right) \cdot \mathbf{P}^{5} & =P_{D} \\
\mathbf{S} \cdot \mathbf{P}^{5} & \leq \mathbf{p}^{\max }
\end{aligned}
$$

Both the solution $\mathbf{P}^{5}$ and the eventual market response to the adders $\beta^{*}$ are identical with the optimal operating point solution $\mathbf{P}^{*}$.

Extension of this method to a nonlinear case involves the use of linearizations around an operating point. 
Variables $\mathbf{P}$ are replaced by $\Delta \mathbf{P}, P_{D}$ is replaced with zero, and the vector $\mathrm{lb}$ becomes all zeros.

An extension of the method to a lossy case would have to comprise two items. First, a loss allocation to the various transactions has to be made, which is possible in principle but which is not treated here. Second, penalty factors applicable to each generator have to be included. Both items can be handled by the ISO, each derived from a power flow and its Jacobian matrix. The ISO adds superimposed quadratic price signals and introduces the penalty factors in the power balance equations 1 as follows:

$$
\alpha_{1} \cdot \Delta P_{1}+\alpha_{2} \cdot \Delta P_{2}+\cdots+\alpha_{n_{g}} \cdot \Delta P_{n_{g}}=0
$$

where the $\alpha$ coefficients are the penalty factors obtained from the transposed Jacobian [15].

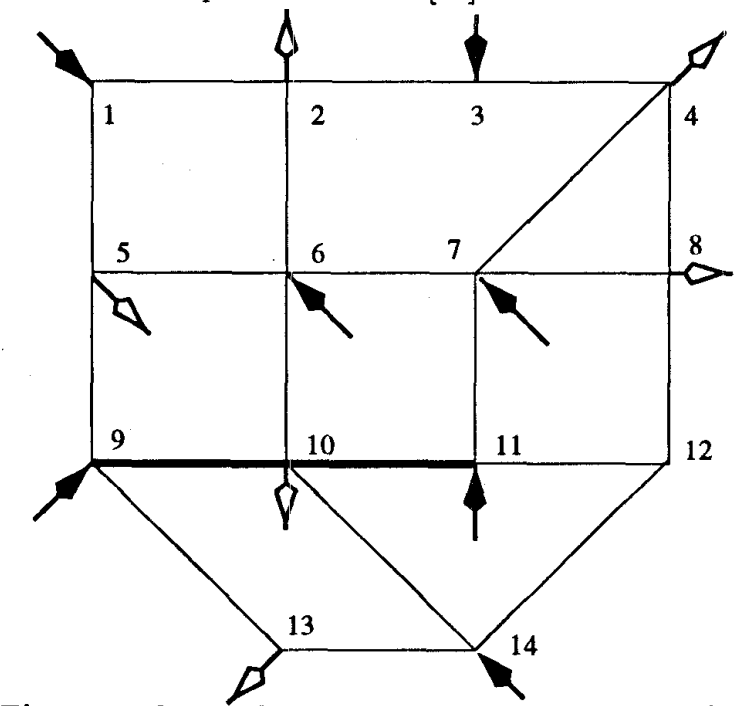

Fig. 1: The 14 bus test system, congested lines are highlighted.

\section{A simulated market behavior experiment}

This section describes a comprehensive simulated experiment and observes the response of the system and the behavior of the generators. The experiment starts by creating a network where the optimum operating point results in transmission grid congestion.

Step 0 (reference): Read all cost, network and constraint information. Solve a constrained OPF problem. Determine the values of optimal congestion price adders and verify that the unconstrained solution with these adders is the same.

Step 1 (market): The experiment begins here. Observe the market under two different unconstrained operating conditions, $\mathrm{P}^{1}$ and $\mathbf{P}^{2}$.

Step 2 (ISO): Guess $\tilde{c}_{1}$, determine $\tilde{c}_{2}, \ldots, \tilde{c}_{n_{g}}$.

Step 3 (ISO): When congestion is detected at $\mathbf{P}^{3}$, solve an OPF problem (solution $\tilde{\mathbf{P}}$ ) and determine additive costs $\beta$ (nodal transmission congestion prices).

Step 4 (market): Issue these prices and allow the market settle into a new operating point $\mathrm{P}^{4}$.

Step 5 (ISO): By observing changes in behavior due to changes in prices, estimate $K$, solve the OPF (solution $\left.\widetilde{\mathbf{P}}^{*}\right)$ and determine new congestion prices $\beta^{*}$.
Step 6 (market): Issue congestion prices $\beta^{*}$ and allow the market to settle into condition $\mathbf{P}^{5}\left(=\mathbf{P}^{*}\right)$.

The system used is the 14 bus 7 generator system illustrated in Figure 1. The results are listed in table 1 and all necessary data to reproduce these results are listed in tables 2,3 and 4 .

The method has also been applied to the 3-bus example in [9] (the same solution is obtained), and, as an incremental method, to a case based on the IEEE 300 bus test system ${ }^{6}$ and to a 1390 bus system with 158 generators and 36 simultaneously congested lines.

\section{Economic aspects of the corrective signals}

Congestion can be managed by nodal prices, where these prices lead to an optimum if all parties behave rationally. However, the value of the congestion adder has still one degree of freedom: any number can be added to all prices and its effect on congestion relief is the same. Two other aspects of congestion that have not been explicitly addressed so far include: the need to use congestion as an economic signal for the need to network expansion, and the handling of contingency constraints in a cost-optimal manner (i.e., a manner that considers the ex-ante expected costs of potential outages). Both these subjects are beyond the main scope of the paper, but brief comments are in order.

Consider the effect of congestion prices on generators. The present paper has sidestepped the issue of revenue generation by congestion pricing by making it possible to have zero-revenue congestion-relieving prices. That is, under congestion some producers benefit while others do not. A producer contemplating the startup of a unit and the submittal of a bid (or even the construction of a unit) must analyze the expected total levels of production of his/her unit and anticipated prices of the market (including payments or income from anticipated congestion) as part of a decision to bid. This analysis must consider ex-ante any risks associated with market and/or congestion uncertainties.

Consider now the effect of congestion on transmission providers. Since transmission providers are monopolistic, they stand to benefit from congestion pricing unless either (1) they are carefully regulated or (2) they are regulated indirectly, by a mechanism that lines up their self-interest with society's interest in lowest cost power production and delivery. This second idea is akin to performance-based regulation. Higher prices during congestion conditions may be desirable to give signals to the loads for reduced congestion. However, if the result is higher revenues for the transmission company during these conditions, the incentive will be there for congestion to remain. This is of particular concern, since for most other commodities congestion leads to a reduction in consumption and the desire for expansion: a company has the incentive to expand capacity to sell more when congestion is reached (and prices are stable). In the case of the transmission company, the case is different: the total power transmitted remains

\footnotetext{
${ }^{6}$ Available from $\mathrm{ft}_{\mathrm{p}}$ ///wahoo.ee washington.edu/.
} 
Table 1: Summary of results of experiment.

\begin{tabular}{|c|c|c|c|c|c|c|c|c|c|}
\hline Step & Name & Notes & Gen 1 & Gen 2 & Gen 3 & Gen 4 & Gen 5 & Gen 6 & Gen 7 \\
\hline Optimal solution & $\overline{\mathbf{P}^{*}}$ & o & 1.2948 & 1.1761 & 0.4995 & 0.7780 & 0.4867 & 0.9431 & 3.2717 \\
\hline 1st market observation & $\mathbf{P}^{1}$ & $m$ & 0.9966 & 0.7832 & 0.0900 & 0.2966 & 0.8725 & 0.7040 & 1.0770 \\
\hline 2nd market observation & $\mathbf{P}^{2}$ & $\mathrm{~m}$ & 1.1023 & 0.8646 & 0.1715 & 0.4023 & 0.9853 & 0.7951 & 1.1889 \\
\hline Unconstrained market & $\mathbf{P}^{3}$ & $c, m$ & 1.5524 & 1.2114 & 0.5188 & 0.8524 & 1.4662 & 1.1831 & 1.6657 \\
\hline OPF with guess for $\tilde{\mathbf{c}}$ & $\widetilde{\mathbf{P}}$ & $\mathrm{i}, \mathrm{o}$ & 1.2948 & 1.1761 & 0.4995 & 0.7780 & 0.4867 & 0.9431 & 3.2717 \\
\hline Market with $\beta$ & $\mathbf{P}^{4}$ & m & 1.5266 & 1.2079 & 0.5169 & 0.8450 & 1.3683 & 1.1591 & 1.8263 \\
\hline OPF with $K \tilde{\mathbf{c}}$ & $\widetilde{\mathbf{P}}$ & i,o & 1.2948 & 1.1761 & 0.4995 & 0.7780 & 0.4867 & 0.9431 & 3.2717 \\
\hline Market with $\beta^{*}$ & $\mathbf{P}^{5}$ & $\mathrm{~m}, \mathrm{o}$ & 1.2948 & 1.1761 & 0.4995 & 0.7780 & 0.4867 & 0.9431 & 3.2717 \\
\hline
\end{tabular}

(o) Optimal solution. The ISO solution for $\widetilde{\mathbf{P}}$ is already optimal.

(m) Solution reached by the market. Market solutions are always unconstrained.

(i) OPF solution calculated by the ISO.

(c) Congested solution.

exactly the same (ignoring the small increase in power due to likely higher losses).

On the subject of ex-ante contingency constraints, the issue is that constraints must not be considered rigid, but they must be weighted against the cost of possible outages that failure to consider a particular constraint must result in. Thus, consideration of contingency constraints based on explicit determination of expected outage costs [16] remains appropriate.

\section{Observations on practicality}

This paper has assumed that market participants are perfectly rational and able to properly and accurately respond to price signals within appropriate time frames. The envisioned scheme uses prices to manage congestion only when markets have time to adjust to these prices. The exact mechanism by which a security coordinator interacts with the market can take many specific forms. One possibility is intervention into the forward market with price signals that are posted for all to use during their trade decisions, with the understanding that these signals, along with the bids, are subject to adjustments following rules similar to those by which the participants are allowed to adjust their bids and trades.

Another practical concern is that even well intentioned players able to respond may fail to react in a predictable, consistent and timely manner in every case. There are considerations other than price that determine the actual behavior of suppliers. The proposals in this paper can (and probably should) be coupled with other means for congestion management that do not rely on market response for congestion relief for more precise congestion relief under such circumstances.

The results of this paper are not dependent on the disclosure of generator costs. They rely instead on price observation to infer cost parameters of suppliers. The approach as described requires "iteration" between suppliers and the ISO. As markets evolve, it is expected that markets that operate under the implicit threat of congestion prices soon learn to negotiate power levels and exchanges that avoid such conditions, and depend on geographically diversified power supply (and demand) portfolios instead.
Linear characteristics (more precisely, constant prices over the whole range of the output of generators) are not compatible with corrective price signals. Hence, nonlinear characteristicvs are essential for pricing to work as described in the paper. This applies to coordinated trading [9] as well. The assumed quadratic price behavior is one example which could be taken as an approximation to a nonlinear or a piecewise linear characteristic. Follow up work has addressed the issue of non-quadratic cost curves. Results illustrate that difficulties arise, but that there exist means to resolve them.

Small adjustment to congestion management pricing signals can be quite effective to manage congestion for units with marginal costs in the vicinity of system marginal costs. When congestion occurs which can only be relieved by units with very low or very high marginal costs, only significant congestion price adjustments will induce these units to respond. The effect is that suppliers with resources at strategic locations within the system will be able to derive significant revenues (or be expected to pay significant prices) during congestion periods without skewing the system-wide marginal cost for uncongested regions ${ }^{7}$. Congestion pricing is thus seen as a practical alternative to capacity rights, whether these rights are defined in a pair-wise manner [1] or as rights on individual lines [2]. It also avoids the unresolved difficulties that capacity rights pose when series flow control devices are introduced into the network.

The notions in this paper are not directly contradictory to capacity rights. They can, in fact, be used to help establish prices for trading of capacity rights. However, the creation of markets that are differentiated in both time and location by means of congestion price signals simplifies trading by allowing the majority of all trading to focus on energy rather than on transmission capacity.

\footnotetext{
${ }^{7}$ It can be shown that a mix of supply units that includes some low investment high marginal cost low duty cycle units can be socially optimal.
} 


\section{Conclusions}

It is possible to use nodal congestion price signals to let power markets attain a societally optimum operating point in the presence of congestion. The solution obtained is identical to that attainable by an Optimum Power Flow. Furthermore, the determination of these prices does not require that the ISO know the actual costs of production of the various generators. The parameters needed (the quadratic coefficient of the cost curves) can be inferred from two passive observations of the market plus one observation of the response of the market to one round of congestion prices. This use of congestion prices is apart from its use as a means for revenue generation or from congestion pricing for ex-ante probabilistic constraints, topics not addressed in this paper.

\section{REFERENCES}

[1] W. W. Hogan, "Contract Networks for Electric Power Transmission," Journal of Regulatory Economics, Vol. 4, 1992, pp. 211-242.

[2] H-P. Chao and S. Peck, "A Market Mechanism for Electric Power Transmission," Journal of Regulatory Economics, Vol 10, pp. 25-60, July 1996.

[3] R. Baldick, R. J. Kaye and F. F. Wu, "Electricity tariffs under imperfect knowledge of participant benefits," IEEE Transactions on Power Systems, Vol. 7, No. 4, pp. 1471-1482, November 1992.

[4] M. C. Caramanis, R. E. Bohn and F. C. Schweppe, "Optimal Spot Pricing, Theory and Practice," IEEE Transactions on Power Apparatus and Systems, Vol. PAS-101, No. 9, Sep. 1982, pp. 3234-3245.

[5] Ralph Masiello. It's put up or shut up for grid controls. IEEE Spectrum, pages 50-51, June 1996.

[6] J. M. Jacobs. Artificial power markets and unintended consequences. Presented at the IEEE/PES Summer Meeting, Denver, Colorado, July 1996, paper 96 581-9 PWRS.

[7] R. Rajaraman, J. Sarlashkar, and F. Alvarado. The effect of demand elasticity on security prices for the Poolco and Multi-lateral contract models. Presented at the IEEE/PES Summer Meeting, Denver, Colorado, July 1996, paper 96 SM 491-1 PWRS.

[8] A. White. The Electricity Industry in England and Wales. James Capel \& Co., February 1990.

[9] Felix F. Wu. Coordinated multilateral trades for electric power networks. In Proceedings of the $12^{\text {th }} P S C C$ Conference, pages 786-792, August 1996.

[10] A joint PSERC-EPRI workshop on underlying technical issues in electricity deregulation, Tucson, Arizona, April 1996. Published by EPRI, 1997.

[11] F. Alvarado, R. Broehm, L. Kirsch, and A. Panvini. Retail pricing of reactive power service. In Proceedings of the 1996 EPRI Conference on Pricing Electric Services, La Jolla, California, USA, March 1996.

[12] R. Bacher and H. Glavitsch. Loss reduction by network switching. IEEE Transactions on Power Systems, PWRS-3(2):447-454, May 1988.

[13] R. Baldick and E. Kahn. Contract paths, phase shifters and efficient electricity trade. Presented at the IEEE/PES Summer Meeting, Denver, Colorado, USA, July 1996, paper 96 SM 495-2 PWRS.

[14] K. Frauendorfer, H. Glavitsch, and R. Bacher, editors. Optimization in Planning and Operation of Electric Power Systems. Springer-Verlag, 1992.

[15] F. L. Alvarado. Penalty factors from Newton's method. IEEE Transactions on Power Apparatus and Systems, PAS-97:2031-2040, Nov/Dec 1978.
[16] F. Alvarado, Y. Hu, D. Ray, R. Stevenson, and E. Cashman. Engineering foundations for the determination of security costs. IEEE Transactions on Power Systems, 6(3):1175-1182, August 1991.

Table 2: Generator cost coefficients.
\begin{tabular}{|r|r|c|c|}
\hline Gen & Bus & b & c \\
\hline 1 & 1 & 85.09 & 0.500 \\
2 & 3 & 85.08 & 0.649 \\
3 & 6 & 85.53 & 0.648 \\
4 & 7 & 85.44 & 0.500 \\
5 & 9 & 85.18 & 0.468 \\
6 & 11 & 85.18 & 0.580 \\
7 & 14 & 85.08 & 0.472 \\
\hline
\end{tabular}

Table 3: Loads. $L_{1}$ and $L_{2}$ correspond to market observations prior to congestion, $L_{3}$ results in congestion.

\begin{tabular}{|r|r|c|c|c|}
\hline Load & Bus & $L_{1}$ & $L_{2}$ & $L_{3}$ \\
\hline 1 & 2 & 1.33 & 1.52 & 1.85 \\
2 & 4 & 0.47 & 0.53 & 0.65 \\
3 & 5 & 0.68 & 0.78 & 0.95 \\
4 & 8 & 0.54 & 0.62 & 0.75 \\
5 & 10 & 1.26 & 1.44 & 3.50 \\
6 & 13 & 0.54 & 0.62 & 0.75 \\
\hline
\end{tabular}

Table 4: Line reactances and line flow limits.

\begin{tabular}{|r|r|r||r|r|r|}
\hline Line & \multicolumn{6}{|c|}{$X$} & $I^{\text {max }}$ & Line & $X$ & $I^{\text {max }}$ \\
\hline $1-2$ & 0.10 & 10.0 & $1-5$ & 0.08 & 10.0 \\
$2-3$ & 0.05 & 10.0 & $2-6$ & 0.05 & 10.0 \\
$3-4$ & 0.10 & 10.0 & $4-7$ & 0.10 & 10.0 \\
$5-6$ & 0.08 & 10.0 & $4-8$ & 0.10 & 10.0 \\
$6-7$ & 0.10 & 10.0 & $5-9$ & 0.08 & 10.0 \\
$7-8$ & 0.10 & 10.0 & $6-10$ & 0.03 & 0.5 \\
$9-10$ & 0.08 & 0.5 & $7-11$ & 0.05 & 10.0 \\
$10-11$ & 0.05 & 1.0 & $8-12$ & 0.08 & 10.0 \\
$11-12$ & 0.10 & 10.0 & $9-13$ & 0.08 & 10.0 \\
$13-14$ & 0.05 & 10.0 & $10-14$ & 0.08 & 10.0 \\
$12-14$ & 0.08 & 10.0 & & & \\
\hline
\end{tabular}

Hans Glavitsch (F'91) received his Diploma of Engineering from the Technical University of Graz, Austria, an MS from Stanford University, California, and a Dr.-Ing. degree from the Technische Hochschule Aachen, Germany. After 17 years with the Brown Boveri Co., he has been appointed professor of electrical engineering at the Swiss Federal Institute of Technology in Zurich, Switzerland. His research interests include power system simulation and control, new concepts for power transmission and system optimization. Fernando L. Alvarado (F'93) obtained a BS degree from the National University of Engineering in Lima, Peru, an MS from Clarkson University, and a Ph.D. from the University of Michigan. He is a Professor at the University of Wisconsin-Madison in the Department of Electrical and Computer Engineering. His main interests are computer applications to power systems, sparse matrix problems and power system security and optimization. 